\title{
El poder del centro: espacios y lugares de la pampa
}

\author{
The Power of the Center: Spaces and Places of the Pampa
}

\author{
Aníbal A. Biglieri \\ biglieri@uky.edu \\ Department of Hispanic Studies \\ University of Kentucky \\ Estados Unidos
}

Recibido: 16/10/2017

Aceptado: 14/1/2018

\begin{abstract}
Resumen
Este artículo trata de la representación de la noción de la pampa en la obra de cinco pintores argentinos y en relación con las descripciones de la región de varios novelistas y poetas argentinos de los siglos XIX, XX y XXI. El análisis se basa en la distinción entre los conceptos de espacios y lugares, y en la forma en que estos últimos se constituyen a partir de un centro de equilibrio (Arnheim, 1982). Los cuadros estudiados son Horizonte (1981) y Pampa, (1981), de Miguel Ocampo; Un potrero (ca. 1900-03), de Ángel Della Valle; Paisaje, de Enrique Policastro; La chacra (1950), de Raúl Soldi; y La tropilla (1954), de Florencio Molina Campos.
\end{abstract}

\section{Palabras clave}

Pintura; literatura: espacios; lugares; centros

\begin{abstract}
This article deals with the representation of the pampa in the work of five Argentinean painters in their relationships with the descriptions of the region by several Argentinean novelists and poets of the $19^{\text {th }}, 20^{\text {th }}$, and $21^{\text {st }}$ centuries. The analysis is based on the distinction between spaces and places and the way in which the latter are constituted from a balancing center (Arnheim, 1982). The paintings studied are the following: Miguel Ocampo's Horizonte (1981) y Pampa, (1981), Ángel Della Valle's Un potrero (ca. 1900-03), Enrique Policastro's Paisaje, Raúl Soldi's La chacra (1950), and Florencio Molina Campos' La tropilla (1954).
\end{abstract}

\section{Keywords}

Paintings; literature; spaces; places; centers 
1 Agradecemos a María Teresa Macario (Facultad de Bellas Artes, Universidad Nacional de La Plata) por la bibliografía que nos hizo llegar, indispensable para la redacción de este artículo.

2 La lectura previa de este texto puede servir para comprender mejor algunas propuestas y marcos conceptuales que serán la base de las páginas que siguen.
Cuando se habla del espacio, se suele pensar en una extensión abstracta y geométrica, homogénea e isomorfa, cuantitativa y mensurable matemáticamente. Según esta concepción euclidiana o cartesiana, el espacio es un vacío independiente de los hechos espacio-temporales a los que les servirá de contenedor. En las ciencias sociales y humanísticas esta idea fue complementada o incluso reemplazada por la de lugar, con múltiples propuestas teóricas y resultados muy diversos, como lo demuestra una bibliografía que, al parecer, no tiene fin (Cresswell, 2004). Pero aunque las teorizaciones sean muchas, variadas y contradictorias, se advierte cierto consenso cuando se trata de distinguir entre espacios y lugares. Brevemente definido, un lugar (place) es un sitio en el cual se despliegan fenómenos y procesos sociales, es decir, es todo espacio (space) en el cual hay una presencia humana explícita u objetos 0 actividades que remiten a una presencia humana implícita aunque no directamente representada (Cresswell, 2004; Paasi, 1991; Tuan, 2007). ${ }^{1}$

Si se considera la bibliografía existente sobre la iconografía de la pampa, todo nuevo estudio al respecto debería empezar por justificarse. En ese sentido, el propósito de este trabajo es estudiar de qué manera en la pintura argentina se representan algunos espacios y lugares de esa región a partir de varios planteos teóricos sobre los espacios abiertos (la pampa bonaerense, la puna jujeña, la meseta castellana, la llanura manchega) llevados a cabo por quien escribe (Biglieri, 2017a 2017b, 2017c, 2016a, 2016b) y a los cuales queda remitido el lector para desarrollos más amplios, precisiones terminológicas y revisión de la bibliografía. El análisis se va a centrar en seis cuadros, pero se mencionarán obras de otros autores, períodos y corrientes estéticas del arte argentino, sin otro fin que el de ampliar el campo de observación, y se trazarán también varios paralelos con textos literarios, especialmente narrativos.

Mediante un método comparativo se estudiarán las relaciones entre imágenes y textos. La selección no sigue un orden cronológico, no tiene preferencia por pintores, por escritores o por escuelas artísticas y literarias ni supone una valoración estética de las obras, sino que se ha hecho nada más que con el fin de ilustrar las premisas básicas sobre los espacios frente a los lugares y sus centros, en una marcha que va desde la literatura hacia la pintura y desde ésta hacia aquélla. Más precisamente, el trabajo es una continuación y un complemento para el texto "Los espacios abiertos de la pampa argentina» (Biglieri, 2017a). ${ }^{2}$ Este artículo se centrará en seis cuadros: Horizonte y Pampa, de Miguel Ocampo [Figuras 1 y 2]; Un potrero, de Ángel Della Valle [Figura 3]; Paisaje, de Enrique Policastro [Figura 4]; La chacra, de Raúl Soldi [Figura 5]; y La tropilla, de Florencio Molina Campos [Figura 6].

El orden de los análisis sigue una progresión que va desde la representación de la pampa como espacio, en las obras de Ocampo y de Molina Campos, hasta la pampa como lugar, con presencia humana implícita o más o menos explícita, en los cuadros de Della Valle, Policastro y Soldi. El de Policastro sirve como nexo entre los del 
primer grupo y los del segundo, en el sentido de que allí se aprecia mejor cuál es el poder que tiene el centro para convertir a la llanura pampeana en un lugar, según las definiciones indicadas antes. La idea de poder del centro se basa en la obra de Rudolf Arnheim (1982) de título homónimo, un abordaje fecundo en postulados teóricos y de método para el estudio de la composición en las artes visuales, en general, y para estos seis cuadros, en particular.

\section{La pampa como espacio}

En una primera visión literaria, la pampa es representada como un espacio abstracto, entendiendo por abstracción el «[...] reducir algo a sus elementos esenciales por medio de un proceso de despojamiento de lo accesorio, de lo circunstancial y lo secundario, de lo que no es específicamente inseparable de ese algo" (Córdova Iturburu, 1971, p. 171). La pampa es considerada una región geográfica reducida a lo que suele pensarse como esencial. Por ello, se la presenta como la conjunción de tierra, de cielo y de horizonte. Así la contempla uno de los personajes de la novela Zogoibi: el dolor de la tierra (1926), de Enrique Larreta: «[...] mirando, con sus grandes ojos dulces, el campo, el horizonte, el cielo" (1960, p. 46), como si eso fuese todo lo que hubiera para ver en la pampa. Y así se la pinta, por ejemplo, en La pampa grande (1908), de Martín Malharro, donde se dan cita las notas más características de esas vastas extensiones, hechas de enormes distancias e inalcanzables lejanías, unidas con un cielo no menos ilimitado por el confín del horizonte. A esos efectos, la pintura de Malharro sugiere un paisaje monótono gracias a una paleta con muy pocas variaciones cromáticas. Y así es también como se describe a la llanura en varios textos de la literatura argentina que tanto insisten en la horizontalidad del relieve pampeano y en su monotonía cromática (Biglieri, 2017a).

Pero si con esta obra de Malharro se está aún en el dominio del realismo y lo figurativo, es en varios cuadros de Ocampo donde el proceso llega hasta sus (casi) últimas consecuencias, como en los acrílicos Pampa (1981 y 2005) y Horizonte (1981)[Figura 1]. En este último, la visión casi abstracta de la llanura se logra con líneas horizontales que parecen prolongarse indefinidamente más allá de los límites materiales del marco.

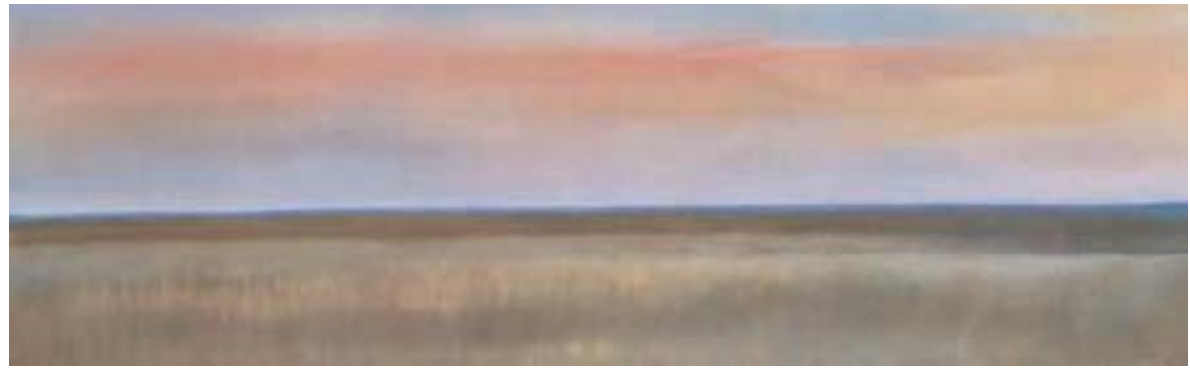

Figura 1. Horizonte (1981), Miguel Ocampo. Acrílico sobre tela, 65 × 200 cm 
En la convergencia de las inmensidades de la llanura y del cielo, la visión se va afinando hacia una imagen plana de la región, hecha de distancias y de la nada. Reducido a varias franjas paralelas, se presenta un espacio pictórico isométrico, homogéneo y uniforme tanto en sus formas como en sus colores, sin ningún atisbo de presencia humana que lo convierta en un lugar. Más aún, la pampa abstracta de Ocampo es ese espacio vacío en el que tanto hincapié hacen novelistas y cuentistas (Biglieri, 2017a), entre ellos Juan José Saer cuando en El río sin orillas (1991) describe las "[...] extensiones interminables de campo vacío" de un "[...] carácter abstracto y geométrico» (1991, p. 63), como si estuviera contemplando también este acrílico. Con terminología de Gilles Deleuze y Félix Guattari (1980), se podría decir que el espacio de Ocampo es un espacio liso (lisse), geométrico, sin accidentes, carente de topografía, como si todos sus puntos fueran iguales. Precisamente, así también se describe la pampa en Los cautivos (2010), de Martín Kohan: «Esas tremendas extensiones, completamente lisas, no contenían nada ni albergaban nada» (p. 44).

Lo mismo ocurre con otras vastas extensiones, como las del cielo o la noche, según las describieron varios escritores argentinos (Biglieri, 2017a). Inmenso también es el cielo pampeano representado por Ocampo en la obra Horizonte (1981) y más aún en Pampa (1981), en el que apenas, muy tenuemente, se divisa un horizonte difuminado y apenas visible en la uniforme oscuridad de la noche [Figura 2]. ${ }^{3}$

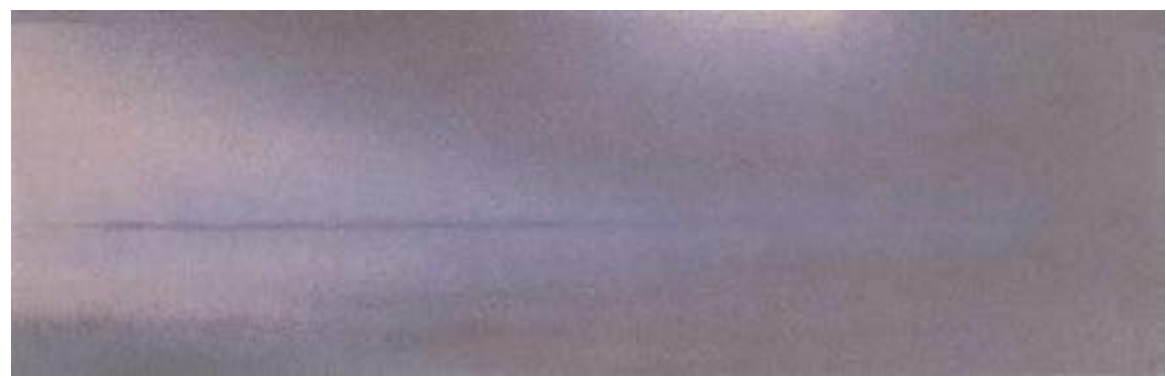

Figura 2. Pampa (1981), Miguel Ocampo. Acrílico sobre tela, 64 × $200 \mathrm{~cm}$

3 La inmensidad del cielo se puede ver también en la pintura de Pío Collivadino Nubes (sin fecha) (Colección Fortabat) y todavía más en Sín título (1976), acrílico sobre tela de Oscar Bony (Malosetti Costa, 2007); también en Se acerca la noche (1994-95), de Luis Felipe Noé. Este cuadro de Ocampo [Figura 2] recuerda algunos pasajes de la literatura argentina, como éste de La cautiva (1837), de Esteban Echeverría: «Noche es el vasto horizonte, / noche el aire, cielo y tierra» (Echeverría, 2006, II, p. 135), o éste otro de Zogoibi de Enrique Larreta, en el cual se describe a la noche pampeana sin luna, ni estrellas y de una «negrura total» (Larreta, 1960, p. 119).

\section{De los espacios a los lugares de la pampa}

La conversión de los espacios en lugares se da de muchas maneras y sería largo inventariar y sistematizar las diversas modalidades con las que ese espacio se humaniza. Por ejemplo, un maizal puede ser un espacio, como sucede en la obra Maizal (1940), de Policastro. También es un espacio el maizal en el cual se encuentran Fabio Cáceres y Aurora en Don Segundo Sombra (1926), de Ricardo Güiraldes (Güiraldes, 1988), pero que se convierte con su presencia en un lugar, según la definición ya mencionada, como un espacio "cercado y humanizado" (Tuan, 2007, p. 54). Como queda dicho, la humanización del espacio puede ser implícita o explícita, pero en todos los casos se entiende como una construcción literaria o 
artística que define a los espacios y a los lugares por la ausencia o por la presencia de seres humanos, respectivamente. En el primer caso, se requiere de la presencia humana; en el segundo, ésta queda más bien sugerida, marcando un primer paso hacia la conversión de un espacio en un lugar. En Zogoibi (1926) la pampa puede ser el ámbito de la soledad: "solitaria llanura interminable», "soledad de los campos» (Larreta, 1960, pp. 9 y 155) y así también es la llanura en Paisaje solitario (1968), de Policastro (Cavanagh, 2005). Pero la llanura no es necesariamente el ámbito de la soledad cuando queda acotada por tranqueras y, alambrados y empieza a poblarse. Según la terminología de Deleuze y Guatari (1980), la pampa se convierte así en un espacio estriado (strié), es decir, cruzado por toda clase de líneas, reales e imaginarias. La pampa se geometriza: "Las vastas extensiones, que hasta entonces permanecieron indivisas, eran rayadas por alambrados, geométricamente extendidos sobre la llanura» (Güiraldes, 1962, p. 125). Dos cuadros ilustran la idea de esta geometrización de la llanura: Monte y tranquera (1974), de Carlos Corotto, y Un potrero (ca. 1900-1903), de Della Valle.

El primero presenta otra imagen de la pampa, ya no se trata de una planicie desnuda y prácticamente desprovista de árboles sino de un monte. Varios escritores aludieron a los montes de la pampa, por citar dos casos, Eugenio Cambaceres en Sin rumbo (1885), o Larreta, en Zogoibi (Larreta, 1960), pero lo importante en este cuadro es notar, en un tercer plano (los dos primeros están ocupados por árboles), una tranquera y un alambrado apenas visibles, signos ya de la presencia humana.

El óleo Un potrero (ea.1900-1903) de Della Valle, es aún más interesante visto desde esta perspectiva del espacio estriado [Figura 3]: el campo pictórico se divide en dos planos, cielo y tierra, unidos y separados por la línea del horizonte - como sucede en La pampa grande (1908), de Malharro; en Horizonte (1981), de Ocampo; o en Paisaje solitario (1968), de Policastro-, pero no son representados solo como espacio, sino también como lugar. En realidad, son tres planos ya que, a diferencia de los otros tres cuadros que se dividen en campo y cielo, en el de Della Valle se delinea una tercera franja situada inmediatamente por debajo de la línea del horizonte. Más aún, toda esta franja intermedia más que estriada horizontalmente, como sucede en el cuadro de Ocampo, está ordenada y cuadriculada en forma de rejilla, es decir, en el sentido en que Arnheim (1982) emplea el concepto de rejilla cartesiana (Cartesian grid) para designar un sistema de líneas rectas que se cruzan en ángulos rectos tanto en una superficie bidimensional como en un espacio tridimensional (Arnheim, 1982).

En el cuadro de Della Valle, entonces, se observa un predominio de las líneas horizontales (horizonte, alambres, palos de la tranquera) y verticales (árboles, palos de la tranquera y del alambrado), en ángulos más o menos rectos. Pero hay ahora un elemento fundamental en la transformación del paisaje pampeano, justamente el que ocupa esa tercera franja intermedia. El espacio tripartito está compuesto por el cielo y por la llanura y ésta, 
a su vez, se divide en dos zonas. Esta división está marcada por el alambrado, que delimita y demarca los establecimientos agrícolas y ganaderos, y por la tranquera, que, en función liminal (del latín limen: umbral), permite el tránsito y la comunicación entre el afuera del campo y el adentro de la propiedad rural (¿una chacra?, ¿una estancia?).

\section{Los alambrados}

Como es de esperar, las novelas de la pampa abundan en referencias a los alambrados y a las tranqueras y no es posible reseñarlas todas, pero sí citar este pasaje de Los caranchos de la Florida, (1916), de Benito Lynch, que hace recordar al cuadro de Della Valle: "[...] una tranquera de rienda, cuyos maderos negros se recortan escuetos sobre el fondo diáfano del horizonte» (Lynch, 1965, p. 134). Omnipresente y ubicuo horizonte, sí, pero aunque no haya ningún ser humano a la vista, en esta llanura ya no «iTodo es cielo y horizonte / en inmenso campo verde!», como se lee en La vuelta de Martín Fierro (1879), de José Hernández (Hernández, 1994, tomo II). No, este paisaje de Della Valle no es solo el espacio de una región natural, sino más bien el lugar de una sociedad y de su historia, porque, como toda región, la llanura es espacio geográfico, tiempo histórico y sociedad humana: en la dilatada geografía de la llanura bonaerense, el alambrado y la tranquera anuncian la chacra o la estancia y, con ellas, una nueva etapa histórica en la organización económica de la sociedad pampeana. Con respecto al alambrado, Noel H. Sbarra sitúa su difusión en la década de 1870 y un «notorio acrecentamiento" a partir de 1875 (2008, p. 82), con lo cual, al terminarse las tierras sin parcelar, el pastoreo (semi)nómade, como el de Don Segundo Sombra (1926), da paso a la explotación agrícola, llevada a cabo dentro de propiedades con límites más o menos bien deslindados y demarcados por los alambrados. ${ }^{4}$

4 Los cuadros Tormenta en la pampa (sin fecha), de Prilidiano Pueyrredón, y $E$ l arado (1901), de Malharro y de Fernando Fader (1915), por ejemplo, registran pictóricamente las labores del campo. Por su parte, Las parvas (la pampa de hoy) (1911), de Malharro, Parvas (1911) y Parvas al sol (1916), de Ramón Silva, y Las parvas (1910), de Eduardo Sívori, documentan la fecundidad de la pampa agrícola.

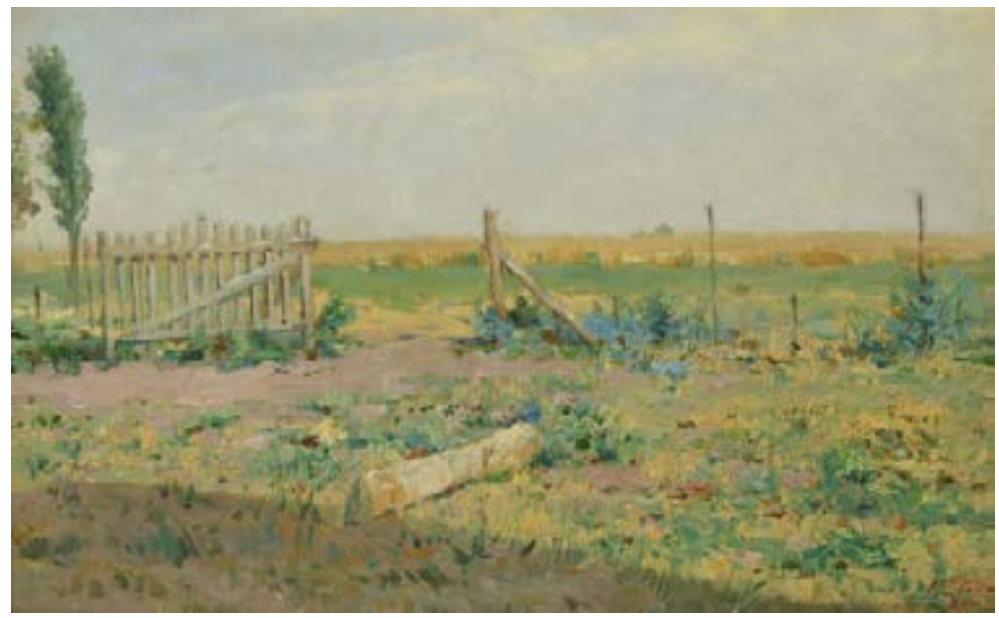

Figura 3. Un potrero (ca. 1900-1903), Ángel Della Valle. Óleo sobre tabla, 23 x $35 \mathrm{~cm}$. Museo Nacional de Bellas Artes 


\section{La pampa y sus centros}

La idea de lugar implica necesariamente la de centro, entendido no en un sentido geométrico o geográfico, sino como punto de convergencia y configurador de los lugares (Biglieri, 2017a). Puede haber centros transitorios (rodeos, carretas, fogones) o más o menos permanentes (rastrilladas, huellas, sepulturas) (Biglieri, 2017a). Sobre ello, intentaremos caracterizar, a continuación, cómo se representaron pictóricamente algunos de esos centros y qué paralelos se pueden establecer con la narrativa argentina. Para comenzar, hay que volver a los cuadros de Malharro, de Ocampo y de Policastro y observar que en ninguno de ellos se halla un centro de convergencia que oriente la mirada del espectador y en torno del cual se congregue un espacio. El análisis del tratamiento del espacio y del lugar en Paisaje, de Policastro [Figura 4], hará comprender mejor en qué consiste el poder del centro - para decirlo con el título del libro de Arnheim, citado anteriormente-

¿Cómo el centro convierte al espacio en un lugar? Para comenzar, habría que volver a Horizonte (1981), de Ocampo [Figura 1], para compararlo después con el de Policastro. Según notó también Jorge López Anaya (2005), y como se confirma en este cuadro, en las obras de Ocampo predominan los esquemas lineales y planos superpuestos o yuxtapuestos y de colores homogéneos, a todo lo cual hay que agregar que, como se dijo previamente, esta llanura es sólo un espacio plano y aplanado, liso y sin puntos de referencia ni perspectiva ni centro que lo transforme en un lugar. Más aún, en este espacio estratificado, las distancias no se representan en recesión, sino que se las yuxtapone en un solo plano: campo, horizonte y cielo no se alejan del observador hacia el fondo, perdiéndose en las distancias, sino que se superponen en dirección ascendente, desde la parte inferior, el campo, hasta la superior, el cielo.

En el cuadro de Ocampo hay un medio (middle), el horizonte, que divide el espacio pictórico en dos mitades pero no un centro (center) en el sentido explicado por Arnheim (1982). En primer lugar, Arnheim distingue entre middley balancing center (centro de equilibrio): el primero indica el medio del espacio pictórico, como es el caso del horizonte en tantos cuadros de la pampa, pero que no es necesariamente el centro de equilibrio (Arnheim, 1982); en otras palabras, el centro de la composición, entendido como el punto de convergencia, de referencia y de anclaje del espacio pictórico, no tiene por qué situarse en el centro geométrico del cuadro: ese centro de equilibrio no implica necesariamente la simetría.

Para aclarar mejor estas ideas se puede volver al cuadro de Della Valle [Figura 3] y preguntarse si hay allí un centro de equilibrio. En Un potrero (ea. 1900-1903) la tranquera sería ese centro y punto de convergencia visual. Pero conviene insistir en que ese espacio no es el centro de equilibrio simplemente por estar en el medio de la franja intermedia, sino porque desde allí se atrae la mirada 
del observador que, pasando por ese espacio abierto, se pierde en la lejanía de campo y cielo. En el cuadro, ese foco está situado entre el primer y el tercer plano, creando así la sensación de la profundidad y la perspectiva que falta en otras pinturas, como las de Ocampo. En ese sentido, se podría concluir que el centro de equilibrio no tiene por qué ser siempre una figura determinada, sino un espacio vacío, un pivote invisible, para decirlo también con terminología de Arnheim (1982).

Con más claridad se aprecia toda esta problemática en el cuadro de Policastro [Figura 4], cuyo centro, representado por un molino de viento, requiere de algunas precisiones más.

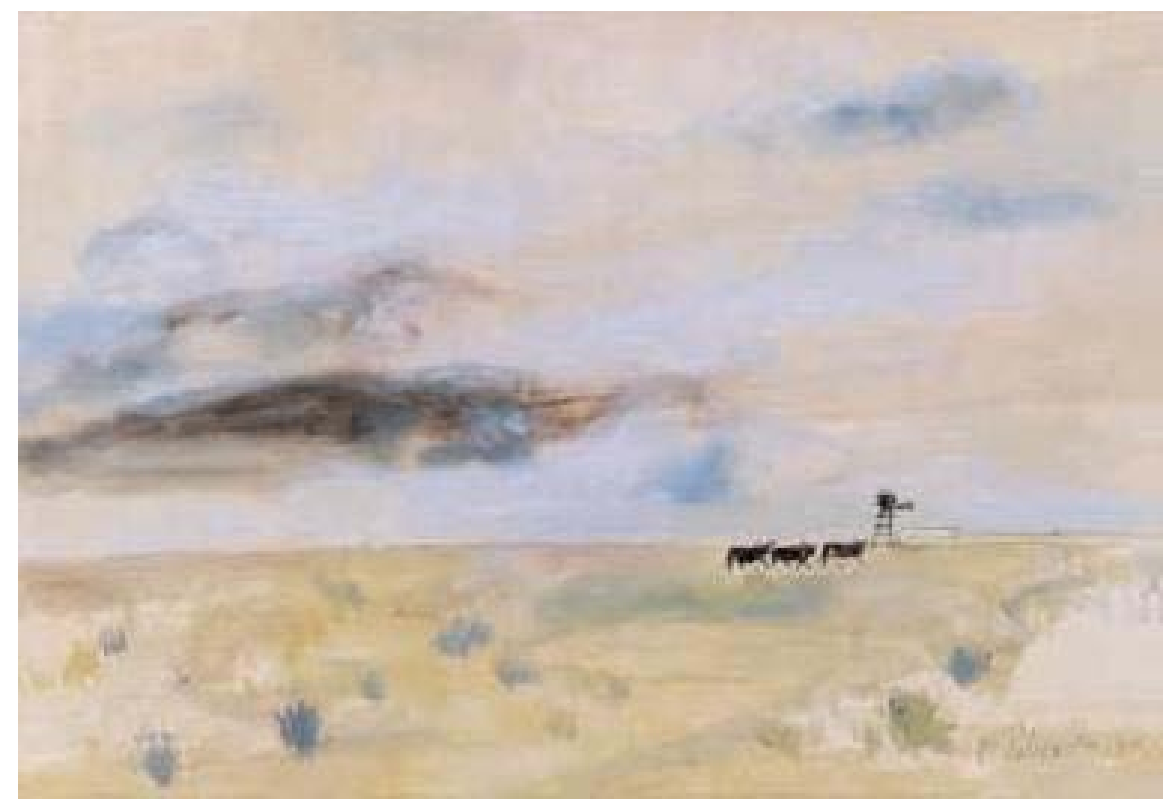

Figura 4. Paisaje, Enrique Policastro. Técnica mixta, 26 × 37 cm

Paisaje también se divide en dos superficies, como Horizonte, La pampa grande, Paisaje solitario y tantos otros cuadros sobre la pampa, pero a diferencia de lo que sucede en todos esos ejemplos, aquí se divisa a la distancia un molino de viento sobre la misma línea del horizonte. Nótese ante todo que si bien este molino no está en el medio del cuadro y no es, por lo tanto, su centro geométrico, sí es su centro de equilibrio, su punto de convergencia. En ese sentido, a él se dirigen tanto los animales como la mirada del espectador que observa desde afuera. Este cuadro tiene, por lo tanto, un centro que contrasta con la horizontalidad del paisaje. Más específicamente, contra esta horizontalidad se destacan mejor la verticalidad del molino (que marca el único lugar en la vastedad del espacio) y las diagonales paralelas en las cuales, en la tierra, se ordenan los arbustos, sobre todo los de la izquierda (desde el punto de vista del observador), que forman una línea imaginaria que se prolonga con la fila de las vacas encaminándose hacia el molino. Son 
líneas, o vectores, que sugieren la recesión de planos hacia atrás: arbustos $\rightarrow$ vacas $\rightarrow$ molino y horizonte $\rightarrow$ cielo. Lo mismo pasa en Un potrero (ea. 1900-1903), de Della Valle [Figura 3], con ese tronco depositado sobre la tierra, en el primer plano, que incluso en un cuadro dominado por una cuadrícula de líneas horizontales y verticales, sugiere la recesión del espacio, hacia un lejano punto de fuga ubicado más allá de los límites del campo pictórico.

Hay que agregar todavía que la existencia de un centro no implica, necesariamente, la de una sola periferia, entendida como el resto del espacio pictórico cuyos puntos estarían todos situados a la misma distancia de ese centro. En algunos casos se puede distinguir entre centro, semiperiferia(s) y periferia, como lo ilustra la obra de Policastro en la que el molino, el ganado y el resto del espacio ocupan estas tres posiciones, respectivamente. Se representa así al espacio tridimensional. Pero en Paisaje se introduce un nuevo elemento, el tiempo en su fluir: frente a la quietud, la intemporalidad y la inmovilidad de Un potrero (ea. 1900-1903), y de los otros cuadros ya comentados, el movimiento y el tiempo quedan sugeridos por Policastro por el desplazamiento de los animales, lo que implica, dicho sea de paso, que la lectura de este cuadro se hace, como la de un texto verbal, de izquierda a derecha. Como se observa, aunque desplazado hacia uno de los lados del espacio visual, el molino de viento se identifica con el centro y ese desplazamiento es compensado por el color de las nubes, en una suerte de contrapunto y equilibrio cromático.

\section{Los molinos de viento}

Tres comparaciones más harán comprender mejor en qué consiste la implantación de un lugar en plena pampa y el poder del centro para lograr dicha implantación. El molino de viento es también tema pictórico en La chacra (1950), de Soldi [Figura 5], pero las diferencias con Paisaje, de Policastro, deben ser destacadas.

En los dos casos, el molino se desplaza hacia la derecha del observador, pero en la pintura de Soldi, donde ocupa también una posición geométricamente ex-céntrica, no es el centro en el sentido ya explicado: no es foco de convergencia espacial y visual. En este cuadro se disciernen tres franjas horizontales (pasto, casa, cielo) y tres verticales (palmera, casa, molino) para formar lo que Arnheim (1982), llama una «rejilla cartesiana» de ángulos (más o menos) rectos, que aquí segmentan el espacio pictórico en nueve subespacios. La casa está en el medio, allí donde se cruzan los ejes horizontal y vertical y cuya intersección corresponde, aproximadamente, a las dos ventanas del piso alto o, si se prefiere, a la línea de conjunción de las dos alas del techo. Sea como fuere, lo que importa hacer notar, otra vez, es que el centro geométrico no coincide con el centro de equilibrio del cuadro; más aún, no se podría determinar con toda certeza dónde reside este centro de convergencia, ya que palmera, casa y molino compiten igualmente para serlo. 


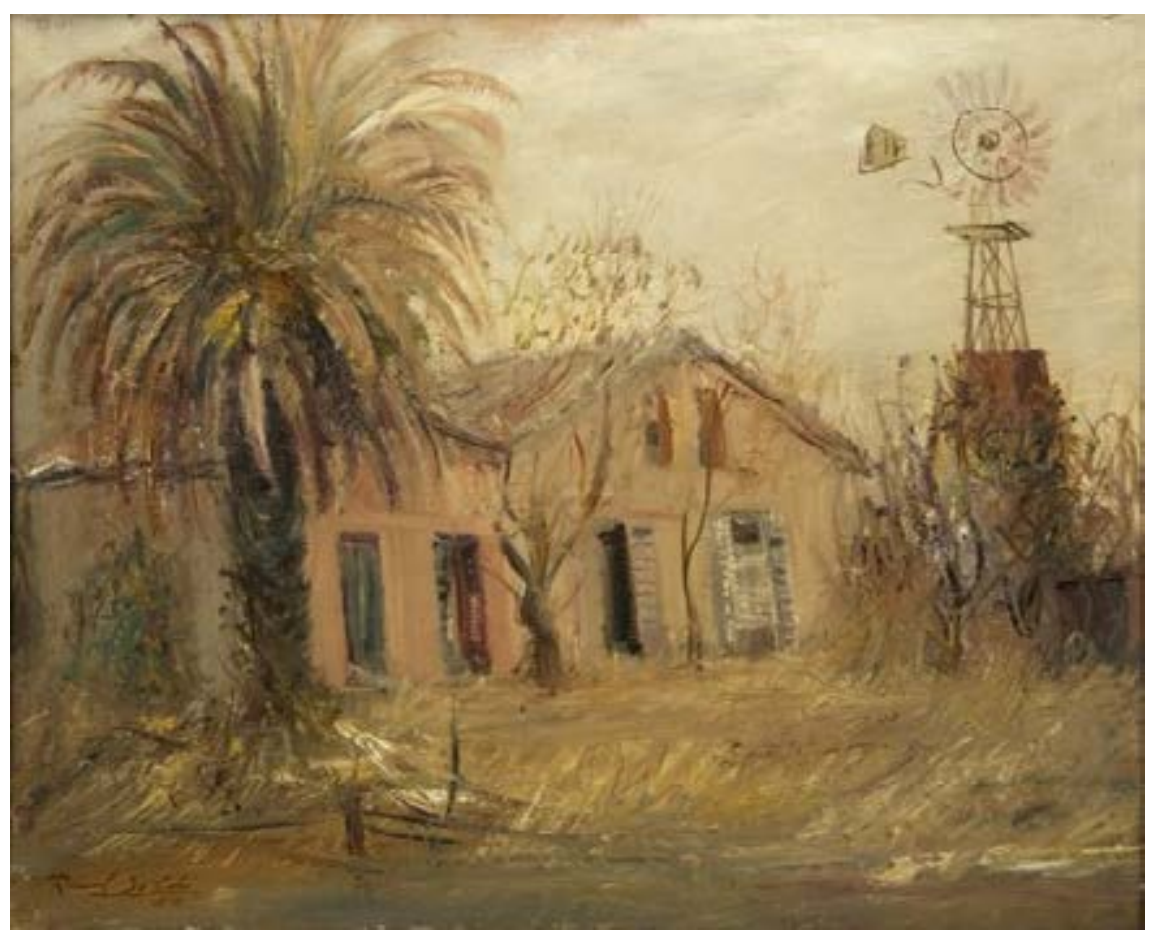

Figura 5. La chacra (1950), Raúl Soldi. Óleo sobre tela, 40 × $50 \mathrm{~cm}$. Colección Fortabat

La comparación entre estas dos pinturas hace ver que la importancia de un elemento, cuando es el centro de equilibrio de un cuadro, no depende ni de sus dimensiones ni del sitio que ocupe en el espacio pictórico. En efecto, los objetos están en función del contexto composicional (Arnheim, 1982): en la chacra de Soldi, el molino es un elemento más; en la inmensidad uniforme de la pampa de Policastro, un diminuto molino se puede convertir en el foco central.

A veces, paradójicamente, el poder del centro se nota aún más cuando está ausente. Para corroborarlo véase ahora La tropilla (1954), de Florencio Molina Campos [Figura 6], cuya composición es la tan habitual y previsible en los cuadros de la pampa: tierra de color más o menos uniforme, cielo cubierto de nubes y un horizonte muy bajo, excepto que aquí se halla, apenas discernible, una tropilla perdida entre las dos inmensidades. Pero no hay un centro de convergencia, un molino, una aguada, un accidente del terreno hacia el cual se dirijan los animales. En el cuadro de Policastro, los animales se desplazan con destino al molino de viento, mientras que en La tropilla no lo hacen en ninguna dirección, precisamente por falta de ese polo de atracción de Don Segundo Sombra (1926), donde la tropilla busca lo que precisamente falta en la pintura de Molina Campos: «Mi tropilla se había alejado caminando con cautela de quien está revisando el campo para comprar, despuntando los pastos, mirando a veces en derredor o a lo lejos, como buscando 
un punto de referencia» (Güiraldes, 1988, p. 261). Pastos, campo y lejanía están también en La tropilla (1954), pero no ese centro que habría orientado la marcha de los animales.

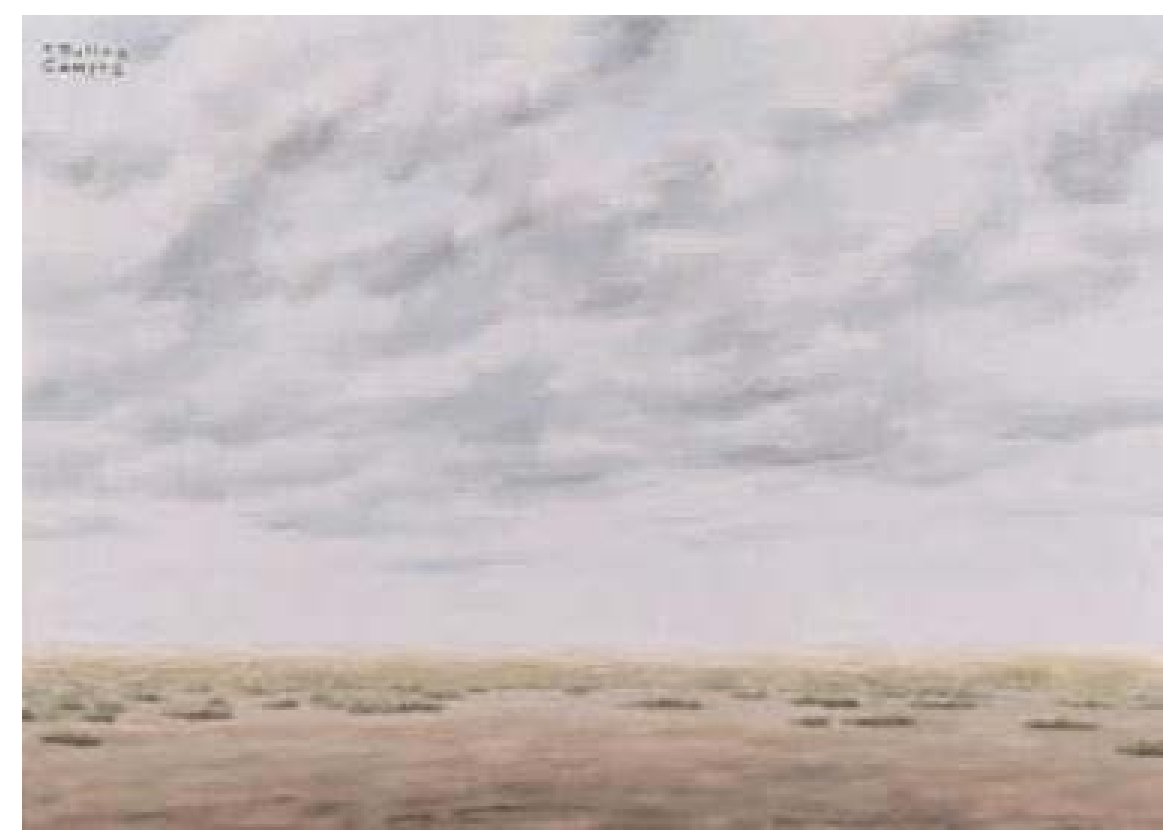

Figura 6. La tropilla (1954), Florencio Molina Campos. Óleo sobre cartón, $36 \times 50,5 \mathrm{~cm}$

\section{Conclusión}

Para concluir, se puede volver a la literatura, punto de partida de este trabajo, cuyo objetivo fue el de ofrecer un análisis comparativo entre cuadros y textos literarios, es decir, como un estudio de las relaciones discursivas intertextuales entre literatura y pintura. $Y$ como último tema de análisis comparativo, hay que recordar ahora varias referencias a los molinos de viento en la narrativa argentina, como en La pasión de los nómades (1994), de María Rosa Lojo, donde la hacienda "[...] bebe de las aguas frescas que acarrean los molinos" (Lojo, 2014, p. 151), o en Los caranchos de la Florida (1916), de Lynch, cuando un caballo bebe y se baña en un molino (Lynch, 1965). Pero los molinos, además de su función utilitaria de dar de beber a los animales, en la novela de Lynch son símbolos de los adelantos que Panchito quiere introducir en la estancia de su padre; en efecto, animado por sus ansias de progreso, arregla un molino y se propone instalar molinos y sistemas de canalización, drenaje y desagües. De allí que un molino que no funcione sirva metonímicamente para describir a una "población que se derrumba bajo el peso del abandono y de los años [...]. Un molino, sin rueda, se hunde, allá bajo, entre la alta maciega, y así, decapitada y escueta, parece su torre el recuerdo melancólico de una civilización que se ha ido» (Lynch, 1965, p. 110). El Molino roto es todo un síntoma de incuria, de 
negligencia y de atraso, que aparece también en Zogoibi (1926): «El molino, a pesar del viento sur que soplaba con fuerza, no funcionaba ¡Qué descuido!» (Larreta, 1960, p. 47). En efecto, esas «torres en la llanura» (Sbarra, 2009, pp. 123-132), junto con el alambrado, el telégrafo y el ferrocarril, transformaron decisivamente el paisaje pampeano con nuevas formas de organización económica y social, ante todo, con la consolidación de las estancias como unidades de producción agrícola y ganadera. A una de ellas hay que volver para concluir, la estancia Albertina de la novela El aromo caído (2012), de Ovidio Lagos, con su molino:

\footnotetext{
Y así salimos al campo abierto, al paso. Apenas ingresé en un lote cubierto de pasturas, clavé los talones en la panza del animal y me lancé, a todo galope, hacia el molino [...] Juntos llegamos al molino y dejamos que los caballos se acercaran al bebedero. El silencio era apenas perturbado por el crujir de las monturas, por el lejano rugido de los novillos: contemplé a Stella, que ya formaba parte del paisaje, de los olores, del sonido. Ella observaba embelesada el imponente casco de Albertina que quebraba la prolija línea del horizonte (Lagos, 2012, p. 169).
}

Siempre el campo abierto y la línea del horizonte, pero ahora a esa línea no la quiebran un monte (Pampa, 1962, de Policastro), un gaucho (Gaucho en la loma, de Tito Saubidet; Cavanagh, 2005), un tambo (El tambo de Quirno, 1873, de Antonio Gazzano) o una pulpería (Tabeando en la pulpería, de Eduardo Sívori), sino el casco de la estancia de una rica familia de terratenientes de la pampa, desierto que es ahora fuente de riqueza y de prosperidad. Y mientras los caballos apagan la sed en el bebedero, los personajes, el molino y la estancia humanizan el espacio con su presencia y lo convierten en un lugar. Enrique Castro comprobará cómo, en ese sitio, Stella ya no está simplemente allí, sino que se ha vuelto parte del paisaje. La pampa se humaniza y, recíprocamente, sus habitantes se identifican con ella.

De acuerdo con lo indicado al principio de este trabajo, el recorrido seguido en los análisis de estas seis obras se propuso mostrar dos concepciones pictóricas de la pampa: primero, como espacio, es decir, como un entorno natural, $y$, segundo, como lugar, como una región geográfica pero también histórica, social y económica. En efecto, la presencia de los alambrados y de los molinos de viento indica la instalación de una sociedad que humanizará el espacio pampeano y lo convertirá en tierras fértiles para la explotación agrícola y ganadera.

\section{Referencias}

Arnheim, R. (1982). The Power of the Center: A Study of Composition in the Visual Arts. Berkeley, Estados Unidos: University of California Press. 
Biglieri, A. (2016a). Castilla como región: reexamen del problema. En J. González del Pozo (ed.). Castilla bajo mirada extraña (pp. 37-58). Valladolid, España: Editorial Páramo.

Biglieri, A. (2016b). La Mancha, de Cervantes al 98. En J. D'Onofrio y C. Gerber (eds.). Don Quijote en Azul: actas selectas de las VIII Jornadas Cervantinas celebradas en Azul (Argentina) en 2015 (pp. 13-37). Tandil, Argentina: Unicen.

Biglieri, A. (2017a). Los espacios abiertos de la pampa argentina. Revista de Teoría de la literatura y Literatura Comparada, (16), 132-157.

Biglieri, A. (2017b). Representación de la pampa argentina en los textos literarios de los siglos XIX a XXI [CD]. Curso dictado en el Pasaje Dardo Rocha, La Plata, Argentina.

Biglieri, AA. (2017c). La Puna de Héctor Tizón: regiones naturales, espacios y lugares. Ponencia dictada en el XI Congreso Argentino de Hispanistas. Universidad Nacional de Jujuy. San Salvador de Jujuy, Argentina.

Cambaceres, E. (1999). Sin rumbo. Madrid, España: Cátedra.

Cavanagh, C. y otros (2005). Vislumbres pampeanas. Buenos Aires, Argentina: EDUCA, Fundación Universitaria Católica Argentina.

Córdova Iturburu, C. (1971). Cómo ver un cuadro: del arte tradicional al cinetismo. Buenos Aires, Argentina: Atlántida.

Cresswell, T. (2004). Place: a Short Introduction. Malden, Estados Unidos: Blackwell Publishing.

Deleuze, G. y Guattari, F. (1980). Capitalisme et schizophrénie 2: milleplateau. Paris, Francia: Les Éditions du minuit.

Echeverría, E. (2006). El matadero. La cautiva. Madrid, España: Cátedra.

Güiraldes, R. (1962). Antítesis. La estancia vieja. En Obras completas (pp. 125-129). Buenos Aires, Argentina: Emecé.

Güiraldes, R. (1988). Don Segundo Sombra. Madrid, España: Colección Archivos.

Hernández, J. (1994). Martín Fierro. Madrid, España: Castalia.

Kohan, M. (2010). Los cautivos. Buenos Aires, Argentina: Debolsillo.

Lagos, O. (2012). El aromo caído. Buenos Aires, Argentina: El Ateneo.

Larreta, E. (1960). «Zogoibi»: el dolor de la tierra. Madrid, España: EspasaCalpe.

Lojo, M.R. (2014). La pasión de los nómades. Buenos Aires, Argentina: Debolsillo.

López Anaya, J. (2005). Arte argentino: Cuatro siglos de historia (16002000). Buenos Aires, Argentina: Emecé.

Lynch, B. (1965). Los caranchos de La Florida. Buenos Aires, Argentina: Troquel.

Malosetti Costa, L. y otros (2007). Pampa, ciudad y suburbio. Buenos Aires, Argentina: Fundación OSDE.

Paasi, A. (1991). Deconstructing Regions: Notes on the Scales of Spatial Life. Environment and Planning A, (23), 239-256.

Saer, J. J. (1991). El río sin orillas. Tratado imaginario. Madrid, España: Alianza. 
Sbarra, N. H. (2008). Historia del alambrado en la Argentina. Buenos Aires, Argentina: Letemendia Casa Editora.

Sbarra, N. H. (2009). Historia de las aguadas y el molino. Buenos Aires, Argentina: Letemendia Casa Editora.

Tuan, Y. (2007). Space and Place: The Perspective of Experience. Minneapolis, Estados Unidos: University of Minnesota Press. 